\title{
Influencia de la formulación de la arena en verde en su difusividad térmica y su efecto en una pieza colada
}

\author{
M.E. Noguez*, I. Beltrán*, T. Robert*, G. Salas* y J. Ramírez* \\ Resumen Se presentan evidencias de que la formulación de una arena en verde (relación entre los \\ porcentajes de agua y de bentonita, a/b, en el rango 0,2-0,55 para 6, 7, 8 y $9 \%$ de \\ bentonita) parece determinar su transferencia de calor y, por tanto, su coeficiente de \\ difusividad térmica, lo que influye en la rapidez de solidificación y en las macroestructuras \\ de piezas coladas en aluminio.
}

Palabras clave Arena en verde. Difusividad térmica. Macroestructuras de colada.

\section{Influence of green sand formulation on thermal diffussivity and its effect on a casting}

\begin{abstract}
Data evidencing a relation among green sand formulations (\% water/\% bentonite, $w / b$, in the ranks $0.2-0.55$ for $6,7,8$ and $9 \%$ bentonite), their heat transfer behaviour, specifically their thermal diffusivity, the casting solidification rate and the resulting macrostructures in aluminium castings are presented.
\end{abstract}

Keywords

Green sand. Thermal diffusivity. Casting macrostructures.

\section{INTRODUCCIÓN}

Se han realizado mediciones de las propiedades térmicas a los materiales de moldeo, con diferentes métodos y a diferentes mezclas, tratando de obtener funciones y coeficientes que se puedan generalizar para su uso ${ }^{[1]}$. Para una arena en verde, es bien conocido el hecho de que al variar el contenido de bentonita y de agua en las mezclas se definen sus propiedades físicas, determinadas por una estructura de unión entre las partículas; parece lógico pensar que las propiedades térmicas respondan también a esas variaciones por lo que no debieran generalizarse los coeficientes. Al cambiar éstos se modifican también la microestructura y propiedades de las piezas coladas ${ }^{[2]}$.

Sin pretender ahondar en el tema, la cohesión entre los granos de arena se debe principalmente a la existencia de capas de agua rígida entre las partículas de bentonita. La relación de agua/bentonita, $a / b$, es el factor dominante ${ }^{[3]}$. Un cierto número de capas de agua rígida coincide con una relación específica de a/b. Para una relación a/b de, aproximadamente, 0,3 se obtiene el menor espaciamiento entre el conjunto bentonita-agua-arena y un mínimo de densidad ${ }^{[4]}$. Mayor cantidad de agua significará un mayor distanciamiento de las fuerzas superficiales ya que hay agua en exceso sobre la rígida; el aglutinamiento disminuye, la densidad aumenta y la unión entre partículas disminuye. Se explica así que otras propiedades mecánicas, como resistencia a la compresión, resistencia a la tracción, compactabilidad, etc., respondan también al óptimo acomodamiento de las capas de agua rígida. En el desarrollo de las resistencias, además de las capas de agua, obviamente hay que considerar el área de contacto entre partículas y la cantidad de puntos de contacto ${ }^{[5]}$. Este estado óptimo de la mezcla se conoce como punto "temper", óptimo o de temple de la arena. Se ha establecido experimentalmente que las diferentes propiedades físicas que responden a esa estructura de temple muestran diferentes grados de dispersión de los valores a/b, variando estos entre 0,22 y $0,38^{[6]}$.

El calor se transmite por conducción, a través de los puntos de contacto de las partículas individuales; por lo tanto, la humedad de la mezcla, que contribuye a la aglutinación, y el grado de

$\left({ }^{*}\right)$ Universidad Nacional Autónoma de México. Departamento de Metalurgia, Facultad de Química, Circuito Institutos s/n, Ciudad Universitaria, C.P. 04510, México, D.F. E-mail: nogueza@servidor.unam.mx 
compactación, que ayuda a obtener los puntos de contacto, afectan la conductividad. Además, en general, ésta aumenta con la temperatura. El efecto de la densidad (ya sea de densidad compactada o densidad a granel) en la conductividad térmica ha sido probado experimentalmente por numerosos investigadores ${ }^{[7}$ y 8$]$. La densidad compactada es la manifestación de una estructura de unión entre partículas que ocasiona valores específicos de propiedades mecánicas, como el máximo en resistencia. Así, debe suponerse que la conductividad tendrá relación también con esta propiedad física ${ }^{[9]}$.

Los datos reportados en la literatura se refieren a conductividad térmica. La difusividad térmica tiene una relación directa con la conductividad a través de la conocida expresión:

$$
\alpha=\frac{k}{\mathrm{C}_{p} \delta}
$$

en donde $\alpha$ representa la difusividad térmica $\left(\mathrm{m}^{2} \mathrm{~s}^{-1}\right) ; k$, la conductividad térmica $\left(\mathrm{kg} \mathrm{m} \mathrm{s}^{-3} \mathrm{~K}^{-1}\right)$; $\mathrm{C}_{p}$, el calor específico $\left(\mathrm{m}^{2} \mathrm{~s}^{-2} \mathrm{~K}^{-1}\right)$ y $\delta$ es la densidad $\left(\mathrm{kg} \mathrm{m}^{-3}\right)$. Como el calor específico y la densidad varían en límites estrechos, lo que se expresa para conductividad térmica puede considerarse válido, cualitativamente al menos, para la difusividad térmica.

En este estudio se miden los coeficientes de difusividad térmica a varias formulaciones de arena en verde, determinadas por la variación de sus relaciones $\mathrm{a} / \mathrm{b}$, con el objeto de evidenciar los efectos de éstas en aquellos y se observa su influencia en una pieza metálica de aluminio solidificada en moldes preparados con dichas formulaciones.

\section{MATERIALES Y PROCEDIMIENTO EXPERIMEN- TAL}

Para las mezclas de moldeo se usaron: arena silícea Oklahoma, de granulometría 100-110 y bentonita sódica (activada) de un proveedor nacional con sede en Veracruz, México. Se prepararon mezclas de $6,7,8$ y $9 \%$ de bentonita, variando la humedad de las mismas en 2, 2,5, 3 y $4 \%$, con lo que se obtuvieron las relaciones de agua/bentonita mostradas en la tabla I. La mezcla de $6 \%$ de bentonita se ensayó con 1,5 \% de humedad para obtener una relación a/b de 0,25 .

Las mezclas se prepararon usando materiales nuevos cada vez, en un molino Simpson de laboratorio con cargas de $3 \mathrm{~kg}$. La molienda se realizó agregando el agua a la arena en la proporción se-
Tabla I. Relaciones agua/bentonita empleadas en la experimentación

Table I. Experimental water/bentonite ratios

\begin{tabular}{lllll}
\hline $\begin{array}{l}\text { Agua } \\
\text { Bentonita }\end{array}$ & $\mathbf{2 \%}$ & $\mathbf{2 . 5 \%}$ & $\mathbf{3 \%}$ & $\mathbf{4 \%}$ \\
\hline $6 \%$ & 0,33 & 0,41 & 0,5 & 0,66 \\
$7 \%$ & 0,28 & 0,36 & 0,42 & 0,57 \\
$8 \%$ & 0,25 & 0,31 & 0,37 & 0,5 \\
$9 \%$ & 0,22 & 0,27 & 0,33 & 0,44 \\
\hline
\end{tabular}

leccionada, por 1,5 min, después se agregó la bentonita correspondiente y se molió otros $1,5 \mathrm{~min}$ más. La carga y tiempo fueron previamente estandarizados como óptimos en el laboratorio.

Las propiedades físicas medidas a las mezclas fueron resistencia a la compresión, al corte, a la tracción, dureza, compactabilidad, permeabilidad, densidad y resistencia al impacto. Para medirlas se usó la probeta normalizada, la máquina universal Dietert para arenas de moldeo y los diversos aditamentos y aparatos calibrados según la American Foundry Society (AFS).

\subsection{Para la medición de difusividad térmica}

Se utilizó un dispositivo que emplea la probeta norma $5 \times 5 \mathrm{~cm}$, obtenida en forma estandarizada con el apisonador Dietert de tres golpes ${ }^{[10]}$. Los termopares usados fueron de chromel-alumel de calibre 28, el registrador Cole Palmer 60648 de tres plumas. De las curvas registradas del calentamiento de la arena al someterse a la fuente de calor del dispositivo se seleccionaron, arbitrariamente, cuatro temperaturas diferentes: $422,460,510$ y $547 \mathrm{~K}$ $\left(149,187,237\right.$ y $274^{\circ} \mathrm{C}$, respectivamente) para resolver la ecuación que plantea el modelo de solución y determinar la difusividad térmica.

\subsection{Para colar la pieza cilíndrica de aluminio con las diferentes mezclas de moldeo}

Se usó un modelo cilíndrico de $10 \mathrm{~cm}$ de longitud y $5 \mathrm{~cm}$ de diámetro. La temperatura de vaciado fue siempre $973 \mathrm{~K}\left(700^{\circ} \mathrm{C}\right)$. Las dimensiones de las cajas de moldeo son: $25 \times 9 \times 5.5 \mathrm{~cm}$, en cada mitad. Se emplearon cargas de aproximadamente $3 \mathrm{~kg}$ de la mezcla de arena preparada para cada molde. La pieza de aluminio se cortó transversalmente y se preparó para un macroataque con un reactivo de $20 \mathrm{~g}$ de cloruro de cobre en $100 \mathrm{ml}$ de 
agua, seguido por otro de 3 partes de ácido nítrico por una de fluorhídrico.

\section{RESULTADOS}

Los resultados mostraron dos comportamientos en los datos: uno, de las mezclas con 6 y 7 \% de bentonita y el otro, de las de 8 y $9 \%$ de bentonita. Se seleccionó la mezcla del $6 \%$ para ilustrar el primer grupo y la de $9 \%$ para el segundo.

Los valores de difusividad térmica obtenidos a las cuatro temperaturas seleccionadas se representaron frente a la relación a/b para 6 y $9 \%$ de bentonita (Figs. 1 y 2 ).

En la figura 2 está claramente definida una región de mínima difusividad térmica, lo que no sucede en la figura 1. De las propiedades determinadas se seleccionaron las resistencias a la compresión y a la tracción como indicadores del temple y se graficó además la densidad. Las figuras 3, 4 y 5 presentan las relaciones entre estas propiedades en función de la relación a/b para las mezclas de 6 y 9 \%. En la mezcla con $6 \%$ de bentonita (Fig. 3) no se llega a determinar la región del temple como el máximo de resistencia. En las figuras 4 y 5 de $9 \%$ de bentonita sí se observa esta región.

Las figuras 6 y 7 muestran las macroestructuras de las probetas cilíndricas coladas en aluminio en moldes preparados con las mezclas de $6 \%$ bentonita y $9 \%$ bentonita, respectivamente. En la figura 6 se observan las macroestructuras con la mezcla de $6 \%$ de bentonita para las relaciones a/b de 0,33

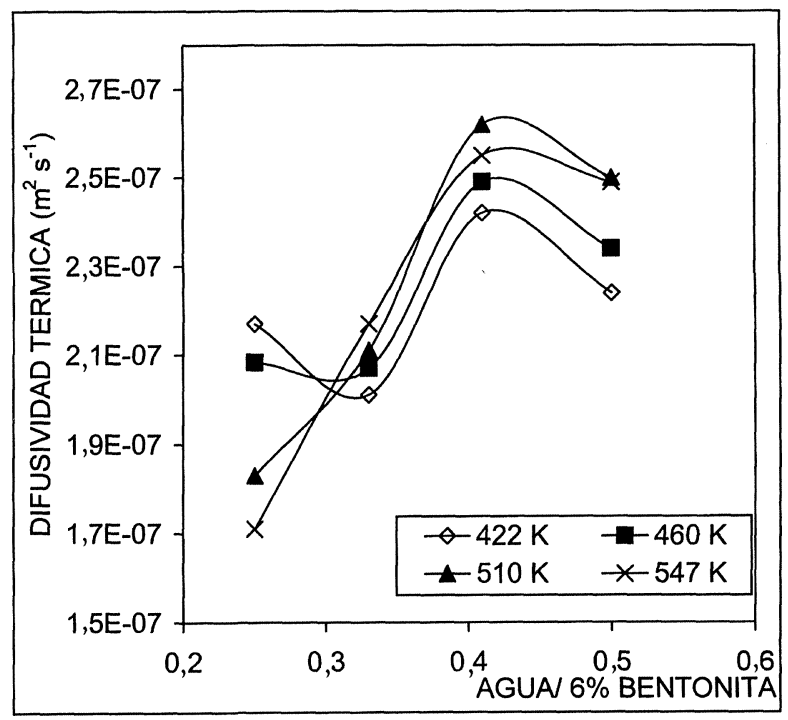

Figura 1. Difusividad térmica frente a la relación \% agua/6\% bentonita.

Figure 1. Thermal diffusivity vs. $\%$ water $/ 6 \%$ bentonite ratio. Rev. Metal. Madrid Vol. Extr. (2005) 143-147



Figura 2. Difusividad térmica frente a la relación \% agua/9 \% bentonita.

Figure 2. Thermal diffusivity vs. \% water $/ 9 \%$ bentonite ratio.

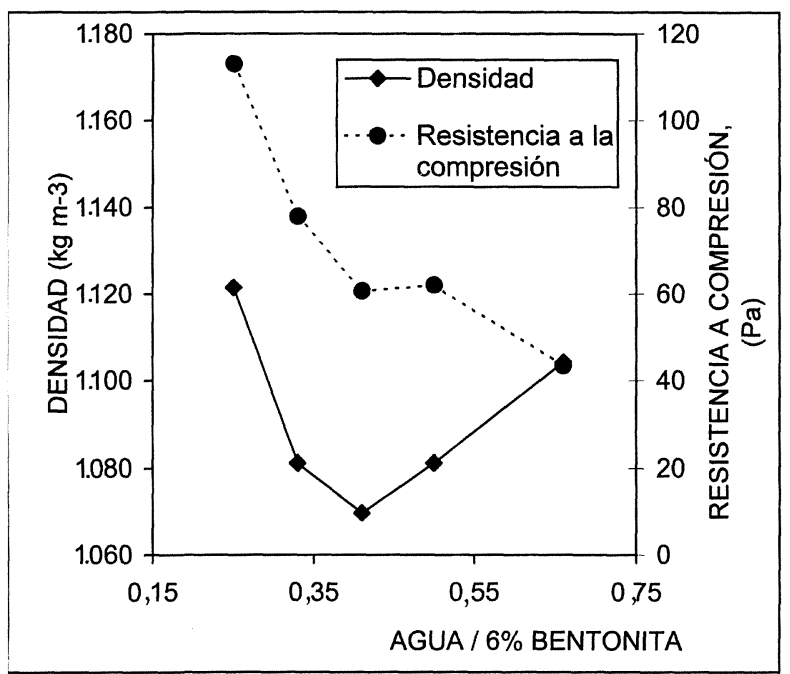

Figura 3. Densidad y resistencia a la compresión frente a la relación $\%$ agua $/ 6 \%$ bentonita.

Figure 3. Densitiy and compressive strength vs. $\%$ water $/ 6$ $\%$ bentonite.

y a/b de 0,5 , mientras que en la figura 7 se observan las de la mezcla de $9 \%$ para cada una de las relaciones a/b empleadas: $0,22,0,27,0,33$ y 0,44 .

\section{DISCUSIÓN}

El primer grupo de mezclas está representado por la de $6 \%$ de bentonita (Figs. 1 y 3 ) y, el otro, por la 


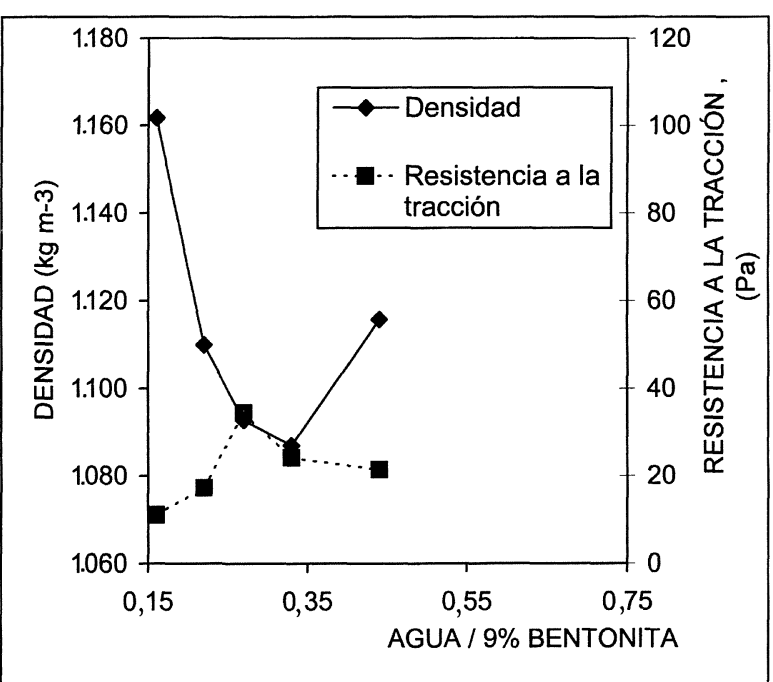

Figura 4. Densidad y resistencia a la tracción frente a la relación \% agua/9 \% bentonita.

Figure 4. Density and tensile strength vs. \% water/9\% bentonite.

de $9 \%$ de bentonita (Figs. 2, 4 y 5). En el primer grupo no se tiene evidencia de un mínimo de difusividad ni de la región del temple. En el segundo, se visualiza muy bien un mínimo de difusividad térmica y de la región del temple, al coincidir los valores de densidad y de resistencia a la tracción y a la compresión en el intervalo a/b de 0,25 a 0,30. Con el objeto de interpretar el significado de los máximos y mínimos en las gráficas, se unió la información de las figuras 3, 4 y 5, de propiedades físicas frente a a/b, con las de las figuras 1 y 2 , de difusividades térmicas frente $\mathrm{a} a / b$. Hay una correspondencia de los valores a/b de la región del temple de las figuras 4 y 5 con los del mínimo de difusividad de la figura 2 . Se puede afirmar que en la mezcla con $9 \%$ de bentonita se presenta una evidencia térmica de la región del temple. Esto es, el punto de mínima densidad y máximo de resistencia a la tracción y compresión, corresponde también a un mínimo de difusividad térmica alrededor de a/b, de 0,27. La relación con la densidad concuerda con lo ya establecido varias veces en la literatura ${ }^{[7}$ y 8$]$, al respecto de que a menor densidad, menor conductividad térmica o, como en este caso, menor difusividad térmica. A relaciones menores al temple, la difusividad vuelve a aumentar así como aumentan los valores de las demás propiedades. La mezcla de $6 \%$ de bentonita empieza con valores bajos de difusividad térmica a la primera relación a/b experimentada, como se puede observar en la figura 1. No hay evidencia de que sea realmente el mínimo ya que no se tienen datos

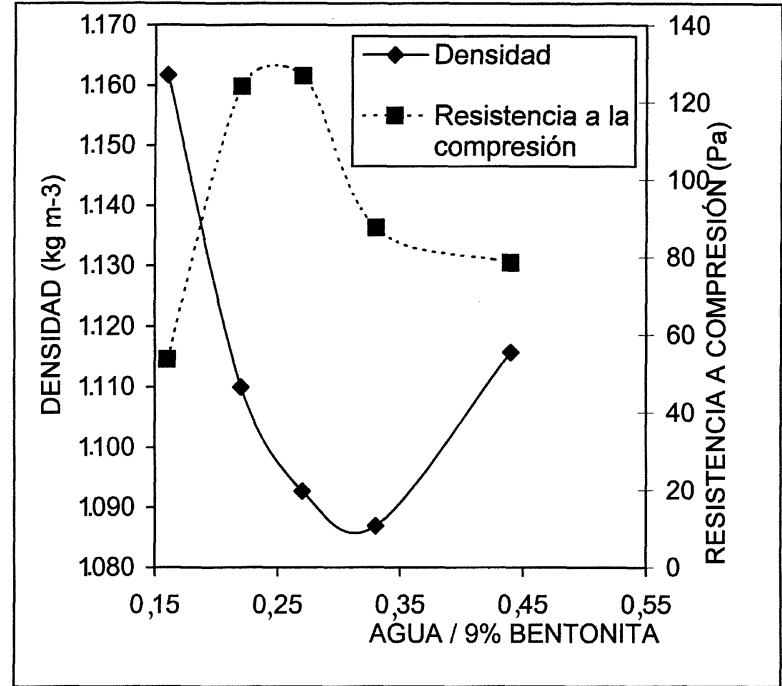

Figura 5. Densidad y resistencia a la compresión frente a la relación $\%$ agua $/ 9 \%$ bentonita.

Figure 5. Density and compressive strength vs. $\%$ water $/ 9 \%$ bentonite ratio.

experimentales a contenidos menores; las mezclas $6 \%$ bentonita son difícilmente manejables para su ensayo con humedades menores al 1,5\%, esto es, a $\mathrm{a} / \mathrm{b}$ menores de 0,25 . Los datos de la resistencia a la compresión de la figura 3 nos indican que, probablemente, no se llegó a ensayar una composición de temple; sin embargo, se puede hablar de una coincidencia en la indefinición del temple, aunque los datos de densidad lo colocan en $\mathrm{a} / \mathrm{b}$ de 0,38 . Con estos datos se evidencia que hay una afectación de la difusividad térmica con la relación $\mathrm{a} / \mathrm{b}$.

Las figuras 6 y 7 muestran las macroestructuras del aluminio colado en las mezclas con 6 y $9 \%$ de bentonita, respectivamente. En la figura 6 se muestran estructuras con diferentes tamaños de grano que corresponden a diferentes velocidades de solidificación provocadas con las mezclas de 6 $\%$ bentonita; la de relación a/b de 0,33 se solidificó más lentamente y, por tanto, el tamaño de grano es ligeramente mayor a la de $a / b$ de 0,5 . Esto concuerda con el valor más bajo de difusividad térmica para esa mezcla. La diferencia de difusividades (leyéndolas para la temperatura de $422 \mathrm{~K}$, $149^{\circ} \mathrm{C}$ ) está entre $2,01 \times 10^{-7} \mathrm{~m}^{2} \mathrm{~s}^{-1}$ y $2,2 \times 10^{-7} \mathrm{~m}^{2}$ $\mathrm{s}^{-1}$, una diferencia aparentemente pequeña pero que se refleja en la estructura. En la figura 7, el mayor tamaño de grano corresponde a la relación a/b de 0,27 que es la de menor difusividad térmica en la figura $2\left(1,9 \times 10^{-7} \mathrm{~m}^{2} \mathrm{~s}^{-1}\right)$ y corresponde a la región del temple en las figuras 4 y 5 . En la figura 


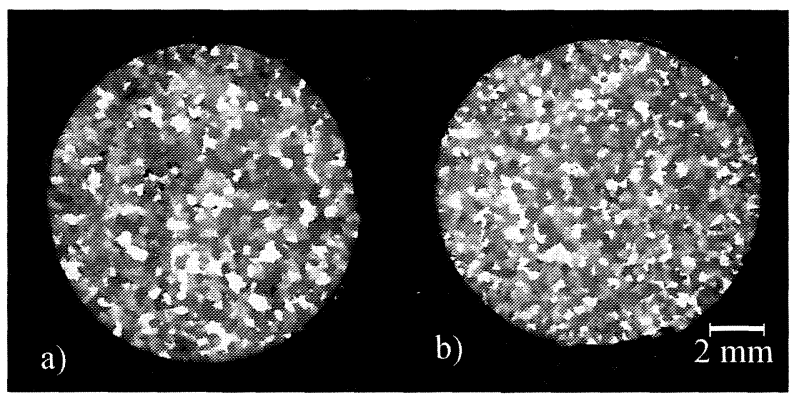

Figura 6. Macroestructuras de aluminio coladas en mezclas de $6 \%$ bentonita: $a$ ) $a / b=0,33 ; b) a / b=0,50$.

Figure 6. Aluminium macrostructures for castings moulded in $6 \%$ bentonite: $a$ ) $a / b=0.33 ; b) a / b=0.50$.

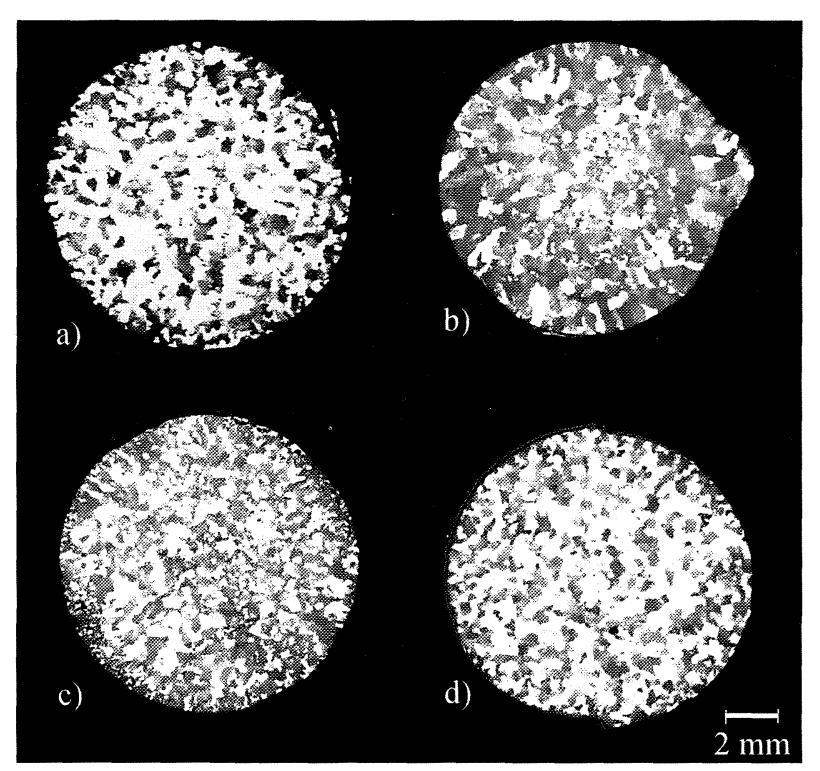

Figura 7. Macroestructuras de piezas de aluminio colado en arena con $9 \%$ bentonita: $a$ ) $a / b=0,22 ; b) a / b=0,27$; c) $a / b=0,33$ y d) $a / b=0,44$.

Figure 7. Sand $9 \%$ bentonite cast aluminium macrostructures: a) $a / b=0.22 ; b) a / b=0.27 ; c) a / b=0.33$ and d) $a / b=0.44$.

7, la relación a/b de 0,22 demuestra claramente que a esta relación se tiene una difusividad térmica alta $\left(2,7 \times 10^{-7} \mathrm{~m}^{2} \mathrm{~s}^{-1}\right)$, por el pequeño tamaño de grano del aluminio colado. Si se piensa que ese punto es un error experimental la macroestructura obtenida demuestra lo contrario. Estos datos corro- boran la correspondencia de datos experimentales de las propiedades físicas y las térmicas, seguramente debidos a la existencia de los puntos de contacto en las partículas de la mezcla.

\section{CONCLUSIONES}

Existen correspondencias entre los valores de $\mathrm{a} / \mathrm{b}$ para los dos grupos de bentonitas que señalan las regiones del temple de las propiedades físicas, los valores mínimos de difusividades térmicas y los cambios en rapidez de solidificación atestiguados por las macroestructuras, por lo que la relación a/b de las mezclas de arena sí afecta la difusividad térmica y, por tanto, la macroestructura de las piezas coladas.

\section{AGRADECIMIENTOS}

A Luis Rodrigo Palomera Rodríguez.

\section{REFERENCIAS}

[1] R.D. Pehlke, A. Jeyarajan y H. Wada, Summary of Thermal Properties for Casting Alloys and Mould Materials, Information Resources National Science, Washington, U. S. A., 1982, pp. 138-163.

[2] J.C. Cuyás, G.W. Mugica, D.O. Tovio y A.C. GonzÁlez, Rev. Metal. Madrid 38 (2002) 139-149.

[3] R.W. Heine, R.A. Green y T.S. Shih, AFS Trans. 98 (1990) 245-252.

[4] AFS Processing and Controlling Clay-Bonded Foundry Sands, Text Course 386, AFS, Illinois, USA, 1970, pp. IV-1 a IV-21.

[5] R.W. Heine y C.W. Florey, AFS Trans. 109 (2001) 901 905.

[6] H.M.D. Roshan, Foundry Trade J. 139 (1975) 333-343.

[7] B.B. RaO y D.C. Williams, Modern Casting 49 (1966) 79-87.

[8] K. Kubo y R.D. Pehlke, AFS Trans. 93 (1985) 405-414.

[9] O.T. FAROUKI, Highway Research Record 128 (1966) 25 44.

[10] I. Beltrán, Tesis Maestría, Facultad de Química, Universidad Nacional Autónoma de México, en proceso. 